\title{
钉镧氧化物涂层析氯反应动力学比较研究
}

\author{
龙 萍 ${ }^{1,2}$, 许立坤 ${ }^{2}$, 崔秀芳 ${ }^{1}$, 金 国 ${ }^{1}$
}

(1. 哈尔滨工程大学 超轻材料与表面技术教育部重点实验室, 哈尔滨 150001; 2. 中国船舶重工集团公司 第七二 五研究所, 海洋腐蚀与防护重点实验室, 青岛 266071)

摘 要: 通过热分解法制备了 $\mathrm{Ti} / \mathrm{RuO}_{2}$ 和 $\mathrm{Ti} / \mathrm{Ru}-\mathrm{La}-\mathrm{O}$ 氧化物涂层, 利用微分电容和极化曲线等实验方法对涂层 $\mathrm{Cl}$ 特性吸附、 $\mathrm{La}$ 对 $\mathrm{RuO}_{2}$ 涂层析氯反应过程的影响及反应机理进行了研究。结果表明, 在 $\mathrm{NaCl}$ 溶液中, 涂层表面存 在 $\mathrm{Cl}^{-}$的特性吸附, 这种特性吸附对析氯反应的动力学产生了影响, 导致 Tafel 斜率上升, 反应级数下降, 加入 $\mathrm{La}$ 使氧化钉涂层的过电位下降，交换电流密度增大，有利于析氯反应的进行。本文对涂层存在的两种可能反应机理进 行了比较分析, 认为在中性 $\mathrm{NaCl}$ 溶液中析氯反应是由 $2 \equiv \mathrm{S} \cdot \mathrm{OCl} * \stackrel{\mathrm{rds}}{\longrightarrow} 2 \equiv \mathrm{S} \cdot \mathrm{O}+\mathrm{Cl}_{2}$ 过程控制的。

关 键 词: 特性吸附; 钉镧氧化物; 析氯反应; Volmer-Tafel 机理; $\mathrm{RuO}_{2}$

中图分类号: TQ174 文献标识码: A

\section{Comparative Study on Kinetics of Chlorine Evolution Reaction for Ru-La-O Oxide Coatings}

\author{
LONG Ping $^{1,2}$, XU Li-Kun ${ }^{2}$, CUI Xiu-Fang ${ }^{1}$, JIN Guo ${ }^{1}$
}

(1. Key Laboratory of Superlight Materials and Surface Technology, Ministry of Education, Harbin Engineering University, Harbin 150001, China; 2. State Key Laboratory for Marine Corrosion and Protection, Luoyang Ship Material Research Institute, Qingdao 266071, China)

\begin{abstract}
The $\mathrm{Ti} / \mathrm{RuO}_{2}$ and $\mathrm{Ti} / \mathrm{Ru}-\mathrm{La}-\mathrm{O}$ oxide coatings were prepared by thermal decomposition of the metal chlorides in the precursor solution. The specific adsorption of $\mathrm{Cl}^{-}$on coatings, the effect of La on the chlorine evolution reactions (ClER) and the kinetic mechanism were investigated by using differential capacity (DC) and polarization curves (PC). Results show that the coating surface exhibits significantly specific adsorption of $\mathrm{Cl}^{-}$in $\mathrm{NaCl}$ neutral solution, which has an influence on the kinetics of the chlorine evolution process, resulting in an increase of the Tafel slope and a decrease of the reaction order. The addition of lanthanum reduces the overpotential of $\mathrm{Ti} / \mathrm{RuO}_{2}$ coating and enhances the exchange current density, which improves the chlorine evolution reaction of the coatings. Both kinetic mechanisms of recombination and electrochemical desorption of adsorbed intermediate species for the coatings are comparatively studied. It is confirmed that the Chlorine evolution reaction on Ru-La-O oxide coatings in $\mathrm{NaCl}$ neutral solution is controlled by the process of $2 \equiv \mathrm{S} \cdot \mathrm{OCl} * \stackrel{\mathrm{rds}}{\longrightarrow} 2 \equiv \mathrm{S} \cdot \mathrm{O}+\mathrm{Cl}_{2}$.
\end{abstract}

Key words: specific adsorption; Ru-La-O oxides; chlorine evolution reaction; Volmer-Tafel mechanism; $\mathrm{RuO}_{2}$

收稿日期: 2014-08-18; 收到修改稿日期：2014-11-06

基金项目: 国家自然科学基金(51275105，51375106); 国家重点基础研究发展计划(973)(2011CB013404); 中央高校基本科 研业务费(HEUCF20151013)

National Natural Science Foundation of China(51275105, 51375106); 973 Program(2011CB013404); Fundamental Research Funds for the Central Universities(HEUCF20151013)

作者简介: 龙 萍(1963-), 女, 博士. E-mail: longping@hrbeu.edu.cn

通讯作者: 金 国, 教授. E-mail: jinguo@hrbeu.edu.cn 
钉基氧化物涂层具有很高的电化学活性, 多年 来在氯碱工业、硫酸盐工业以及水电解、超电容、 燃料电池等领域都受到极大关注。尽管人们对 $\mathrm{RuO}_{2}$ 涂层的析氯反应机理做了大量工作 ${ }^{[1-2]}$, 但所得到 的数据却存在较大的分歧, 例如 Tafel 斜率就有 $120^{[3]} 、 108^{[4]} 、 40 \sim 50^{[5]} 、 40^{[6-7]} 、 31 \sim 32^{[8]} 、 38^{[8]}$ 和 $30 \mathrm{mV}^{[9]}$ 等多种结果, 这种差异可能与阳极的制备 工艺、表面吸附及氧化物表面状态等因素有关 ${ }^{[10-11]}$ 。 由于反应机理的确证主要以动力学参数 Tafel 斜率 和氯离子反应级数为依据, 因此到目前为止, 对钉 基氧化物涂层析氯反应机理还没有获得一致认识。

已提出的析氯反应机理, 有 Volmer-Tafel(再结 合)机理 ${ }^{[12]} 、$ Volmer-Heyrovsky(放电解析)机 理 $^{[5]}$ 、 Kristalik 机理 ${ }^{[13]}$ 以及阳极催化活性物参加反应的机 理 ${ }^{[14-15]}$ 等等, 其中 Volmer-Tafel 机理及 VolmerHeyrovsky 机理被许多研究者证实并用于解释各自 的实验现象。Volmer-Tafel 机理认为, 在电极表面发 生了如下反应:

$$
\begin{gathered}
\equiv \mathrm{S} \cdot \mathrm{O}+\mathrm{Cl}^{-} \rightleftharpoons \equiv \mathrm{S} \cdot \mathrm{OCl}^{*}+\mathrm{e} \\
2 \equiv \mathrm{S} \cdot \mathrm{OCl}^{*} \stackrel{\text { rds }}{\longrightarrow} 2 \equiv \mathrm{S} \cdot \mathrm{O}+\mathrm{Cl}_{2}
\end{gathered}
$$

其中 $\equiv \mathrm{S}$ 为表面活性点, $\mathrm{Cl} *$ 为吸附氯, 第二步是两个 吸附 $\mathrm{Cl}$ *再结合释放的过程, 为速度控制步骤(rds)。而 对于 Volmer-Heyrovsky 机理, 第二步发生如下反应

$$
\equiv \mathrm{S} \cdot \mathrm{OCl}^{*}+\mathrm{Cl}^{-} \stackrel{\mathrm{rds}}{\longrightarrow} \equiv \mathrm{S} \cdot \mathrm{O}+\mathrm{Cl}_{2}+\mathrm{e}
$$

该步骤是析氯反应的速度控制步骤。这两个机理与 表面 $\mathrm{Cl}^{*}$ 覆盖度有很大的关系。当表面 $\mathrm{Cl}$ *覆盖度较 高时, 一般发生再结合过程; 而当覆盖度较低时, 一般可能发生放电解析过程 ${ }^{[16]}$ 。

在 $\mathrm{RuO}_{2}$ 氧化物电极中添加其他元素, 可以提 高其催化活性, 降低成本 ${ }^{[17-19]}$ 。有研究表明, 稀土元 素能够有效提高钓基氧化物涂层的催化活性 ${ }^{[20-21]}$, 其中 La 对电化学活性的影响最为明显 ${ }^{[22]}$ 。但到目 前为止, 关于镧对钉基氧化物涂层析氯过程的动力 学影响还未见报道。在前期对钓镧氧化物涂层成分、 表面结构、双电层界面及电化学活性等研究的基础 上 ${ }^{[23-25]}$, 本工作利用极化曲线、微分电容等实验技术 对该体系涂层的析氯反应机理作了进一步研究, 为钉 镧基系列氧化物涂层的研究提供实验和理论依据。

\section{1 实验方法}

\section{1 电极制备}

将前驱物氯化钉 $\left(\mathrm{RuCI}_{3} \cdot 3 \mathrm{H}_{2} \mathrm{O}\right.$, 上海贺利氏工 业技术材料有限公司, 分析纯)和氯化镧 $\left(\mathrm{LaCl}_{3} \cdot 7 \mathrm{H}_{2} \mathrm{O}\right.$, 分析纯)分别溶解于异丙醇 $\left(\mathrm{CH}_{3} \mathrm{CH}(\mathrm{OH}) \mathrm{CH}_{3}\right.$, 分析
纯)和乙醇 $\left(\mathrm{CH}_{3} \mathrm{CH}_{2} \mathrm{OH}\right.$, 分析纯)溶液中, 然后用摚 拌器将二者按一定比例(Ru: $\mathrm{La}=7$ : 3)混合均匀, 配 置成涂液。

将 TA2 钛基材 $(100 \mathrm{~mm} \times 100 \mathrm{~mm} \times 1.5 \mathrm{~mm})$, 经 轧制、喷砂和碱洗后, 放入 $10 \%$ 沸腾的草酸溶液中 刻蚀 $2 \mathrm{~h}$, 然后将其放入装有 $80^{\circ} \mathrm{C}$ 去离子水的超声 波清洗仪中清洗 $15 \mathrm{~min}$, 烘干。将配制好的涂液均 匀涂刷在刻蚀后钛板的两面, 放入烘干箱中 $120^{\circ} \mathrm{C}$ 烘干 $10 \mathrm{~min}$, 然后送入箱式电阻炉中 $450^{\circ} \mathrm{C}$ 烧结 $10 \mathrm{~min}$, 再进行涂刷、烘干和烧结处理, 反复数遍, 直至总氧 化物载量达到约 $10 \mathrm{~g} / \mathrm{m}^{2}$, 停止涂刷, 最后在箱式电 阻炉中 $450^{\circ} \mathrm{C}$ 烧结 $1 \mathrm{~h}$, 退火。

采用日本 JSM-6700F 场发射扫描电子显微镜 (SEM)自带能谱仪(EDS)对涂层进行了成分分析，分 析结果表明, Ru-La-O 氧化物涂层中 $\mathrm{Ru}$ 含量为 $63.93 \mathrm{at} \%, \mathrm{La}$ 含量为 $36.07 \mathrm{at} \%$, 与设计成分接近, 如 图 1 所示。

\section{2 电化学测试}

利用德国 IM6 电化学工作站进行极化曲线和微 分电容测试。采用经典三电极体系, EG\&G 标准电 解池, 工作电极即上述氧化物阳极, 其暴露工作面 积为 $1 \mathrm{~cm}^{2}$, 辅助电极为铂片, 参比电极为饱和甘录 电极(SCE), 以饱和 $\mathrm{KCl}$ 鲁金毛细管作盐桥, 极化曲 线扫描速度为 $1 \mathrm{mV} / \mathrm{s}$, 电解质溶液取浓度分别为 2 、 3、 4 和 $5 \mathrm{~mol} / \mathrm{L}$ 的 $\mathrm{NaCl}$ 溶液。在电位范围为 $-1.6 \sim 0.8 \mathrm{~V}$, 测试频率为 $50 \mathrm{mHz}$, 步宽为 $5 \mathrm{mV}$, 间 隔 $20 \mathrm{~s}$ 下测试微分电容, 电解质溶液取浓度分别为 $0.001 、 0.01$ 及 $0.1 \mathrm{~mol} / \mathrm{L}$ 的 $\mathrm{NaCl}$ 溶液。

\section{2 结果与讨论}

\section{1 反应动力学常数}

在 Volmer-Tafel 机理中(反应式(1、2)), 第一步 为 $\mathrm{Cl}^{-}$在电极表面放电反应, 形成化学吸附氯, 在这 个过程中 $\equiv \mathrm{S}\left(\mathrm{Ru}^{n+} \rightarrow \mathrm{Ru}^{n+1}\right)$ 被氧化或部分被氧化。第

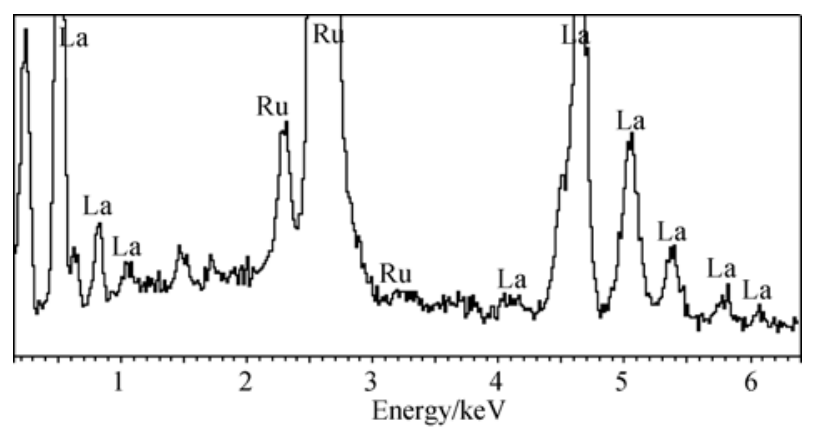

图 1 Ru-La-O 氧化物涂层能谱

Fig. 1 EDS spectra of the Ru-La-O oxide coating 
二步为两个吸附氯再结合, 脱离表面释放 $\mathrm{Cl}_{2}$, 在 Frumkin 吸附条件下, 其动力学方程为 ${ }^{[16]}$ :

$$
i_{2}=2 F k_{2} \theta_{\mathrm{Cl}}^{2}
$$

其中 $k_{2}$ 为反应(2)速度常数, $\theta_{\mathrm{Cl}} *$ 为吸附 $\mathrm{Cl} *$ 的覆盖度, 当反应(1)为准平衡时, 则有:

$$
\theta_{\mathrm{Cl}^{*}}=\frac{F K_{1} c_{\mathrm{Cl}^{-}} \exp (\eta F / \mathrm{R} T)}{1+K_{1} c_{\mathrm{Cl}^{-}} \exp (\eta F / \mathrm{R} T)}
$$

其中 $K_{1}=k_{1} / k_{-1}$ 为反应(1)的平衡常数, 将其代入(4) 式中，则有

$$
i_{2}=2 F k_{2}\left[\frac{K_{1} c_{\mathrm{Cl}^{-}} \exp (\eta F / \mathrm{R} T)}{1+K_{1} c_{\mathrm{Cl}^{-}} \exp (\eta F / \mathrm{R} T)}\right]^{2}
$$

变换方程(6)的形式, 则可得到

$$
\frac{\exp (\eta F / \mathrm{R} T)}{i_{2}^{1 / 2}}=\frac{1}{\left(2 F k_{2}\right)^{1 / 2} K_{1} c_{\mathrm{Cl}^{-}}}+\frac{\exp (\eta F / \mathrm{R} T)}{\left(2 F k_{2}\right)^{1 / 2}}
$$

利用方程(7)绘制 $\exp (\eta F / \mathrm{R} T) / i_{2}^{1 / 2} \sim \exp (\eta F / \mathrm{R} T)$ 关系曲线应该是一条直线。图 2 是将 $\mathrm{Ti} / \mathrm{RuO}_{2}$ 和 $\mathrm{Ti} / \mathrm{Ru}-\mathrm{La}-\mathrm{O}$ 实验数据代入(7)得到的关系曲线, 从 图中可以看到 $\exp (\eta F / \mathrm{RT}) / i_{2}{ }^{1 / 2}$ 与 $\exp (\eta F / \mathrm{RT})$ 均成很 好的线性关系，这表明两种涂层都可能是通过再结 合控制步骤实现析氯反应过程的。通过该曲线可
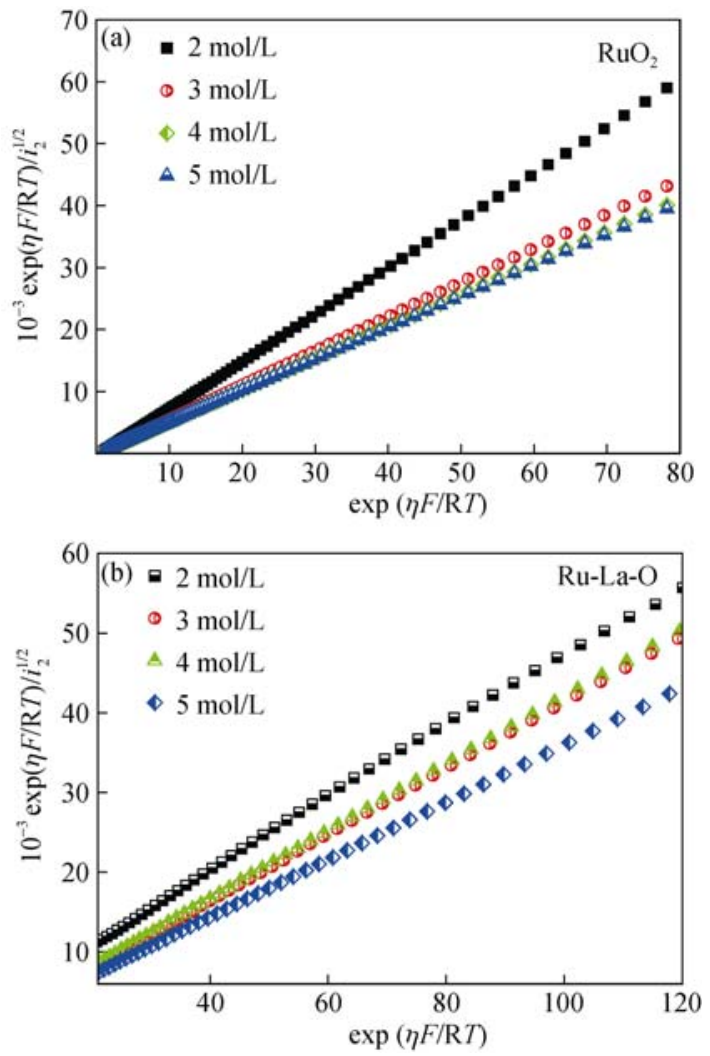

图 2 根据 Volmer-Tafel 机理的公式(7)测试不同浓度下的关 系曲线

Fig. 2 Test of Volmer-Tafel mechanism according to Eq.(7) for various $\mathrm{NaCl}$ solution from 2 to $5 \mathrm{~mol} / \mathrm{L}$
以得到反应的动力学常数 $k_{2}$ 和 $K_{1}$ 。

图 3 为 $k_{2}$ 和 $K_{1}$ 随溶液 $\mathrm{Cl}$ 浓度变化的曲线, 从 图中可以看到，随着溶液浓度增大，反应 $k_{2}$ 明显增 大，这与表面吸附 $\mathrm{Cl}$ *增加有关。而 Ti/Ru-La-O 的 $k_{2}$ 明显大于 $\mathrm{RuO}_{2}$, 表明前者 $\mathrm{Cl}^{*}$ 的覆盖度高于后者。

\section{$2.2 \mathrm{Cl}^{-}$的特性吸附}

氧化物涂层的微分电容是在不发生析氯反应的 电位区间测量的数据，曲线对应最小电位值为 pzc。 由于氧化物涂层表面粗䊁多孔，因此其 pzc 对应的 电容值要比光滑的金属表面电容高很多 ${ }^{[26]}$ 。从图 4 可以看到，随着溶液浓度的增加，pzc 向负偏移，这 表明氯离子显著影响了双电层的结构, 这是因为在 单一电解质溶液中阴离子比阳离子更容易靠近电极 表面, 因此导致 pzc 随着氯离子浓度增加而向负方向 偏移。巴德等认为, 此电位偏移标志着特性吸附 ${ }^{[27-29]}$ 。 此外, 我们还注意到随着电位不同，在最小值两端 吸附/解析的电位也不同, 这是由于在电解质表面存 在 $\mathrm{Cl}^{-}$和 $\mathrm{OH}^{-}$的竞争性吸附所致 ${ }^{[30]}$ 。根据文献[28] 表面 $\mathrm{OH}$ 可能经历如下反应:

$\equiv \mathrm{S}-\mathrm{OH}_{2}^{+} \stackrel{\mathrm{H}^{+}}{ } \equiv \mathrm{S}-\mathrm{OH} \stackrel{\mathrm{OH}^{-}}{\longrightarrow} \equiv \mathrm{S}-\mathrm{O}+\mathrm{H}_{2} \mathrm{O}(8)$ 随着电位增加, 电容增大并逐渐达到最大值, 这是
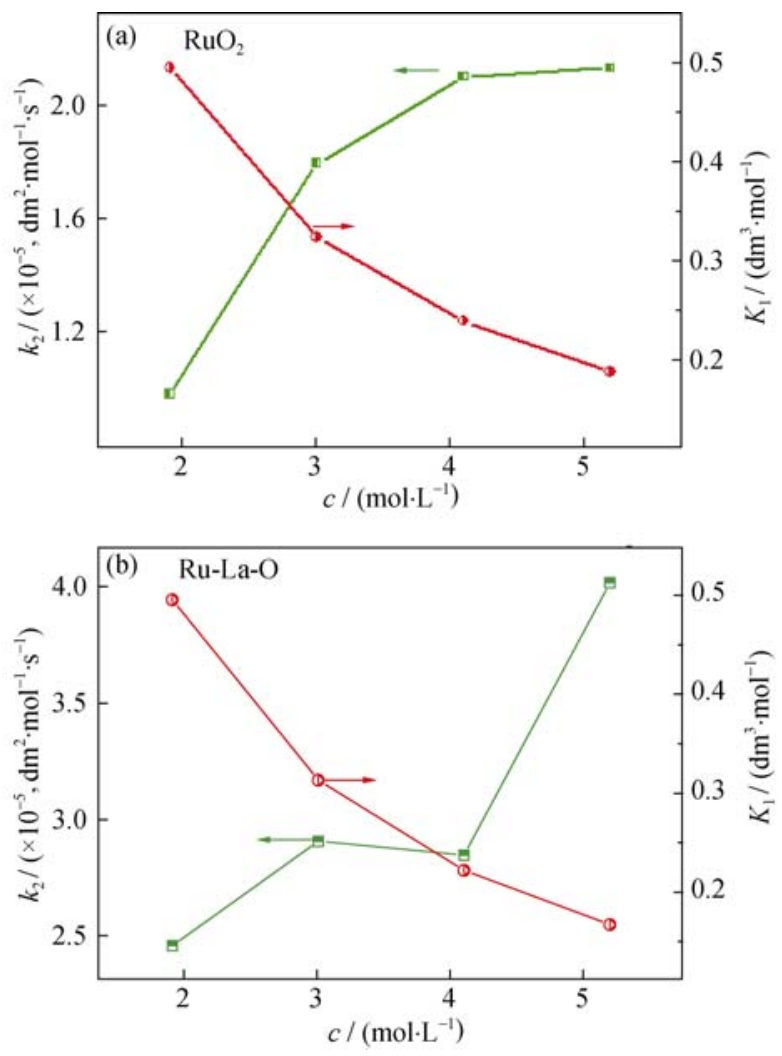

图 3 Volmer-Tafel 机理动力学常数 $K_{1}$ 和 $k_{2}$ 与溶液中氯离子 浓度关系图

Fig. 3 Values of the kinetic constants $K_{1}$ and $k_{2}$ for the VolmerTafel mechanism as a function of chloride concentration in solution 
由于随着表面碱性点的增多, 更有利于 $\mathrm{Cl}^{-}$的吸附, 即发生如下吸附过程:

$$
\equiv \mathrm{S}-\mathrm{OH}_{2}^{+}+\mathrm{Cl}^{-} \rightarrow \equiv \mathrm{S}-\mathrm{OH}_{2}^{+} \mathrm{Cl}^{-} \rightarrow \equiv \mathrm{S}-\mathrm{Cl}+\mathrm{H}_{2} \mathrm{O}(9)
$$

根据实验结果, 随着 $\mathrm{Cl}$ 浓度增加, pzc 负移且 电容明显增大，表明特性吸附的存在以及特性吸附 对表面氧化层电荷的影响 ${ }^{[29]}$ 。与纯 $\mathrm{RuO}_{2}$ 比较 (见图 5), Ti/Ru-La-O 的 pzc 及最大吸附峰显示出更明显 的负偏移(见图 4(a)), 显然加入 $\mathrm{La}$ 明显增强了 $\mathrm{Cl}^{-}$ 的特性吸附作用。Takasu 等 ${ }^{[31]}$ 研究发现, 在 $\mathrm{Ru}$ 基 电极中加入 $\mathrm{La}$, 能够在 $\mathrm{RuO}_{2}$ 表面产生多孔结构, 这必然造成表面活性点数目的增多，也因此提高了表 面碱性点的数目, 从而对表面 $\mathrm{Cl}^{-}$有更大吸附作用, 这 应该是加入 $\mathrm{La}$ 能有效提高 $\mathrm{Cl}$ 特性吸附的主要原因。
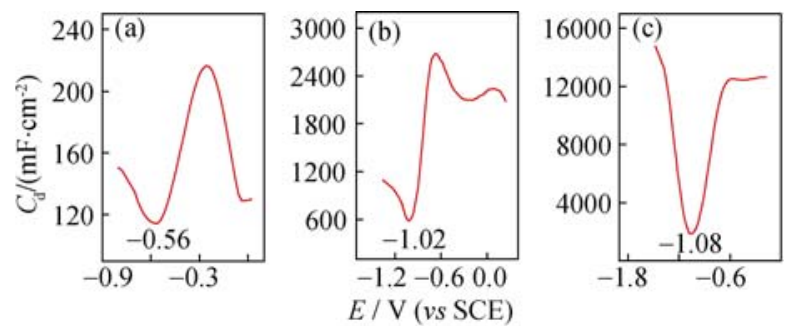

图 4 Ru-La-O 氧化物涂层在(a) 0.001 (b) 0.01 和(c) $0.1 \mathrm{~mol} / \mathrm{L}$ $\mathrm{NaCl}$ 溶液中的微分电容曲线

Fig. 4 Differential capacity curves of Ti/Ru-La-O oxide coatings in (a) 0.001 , (b) 0.01 , and (c) $0.1 \mathrm{~mol} / \mathrm{L} \mathrm{NaCl}$ solution

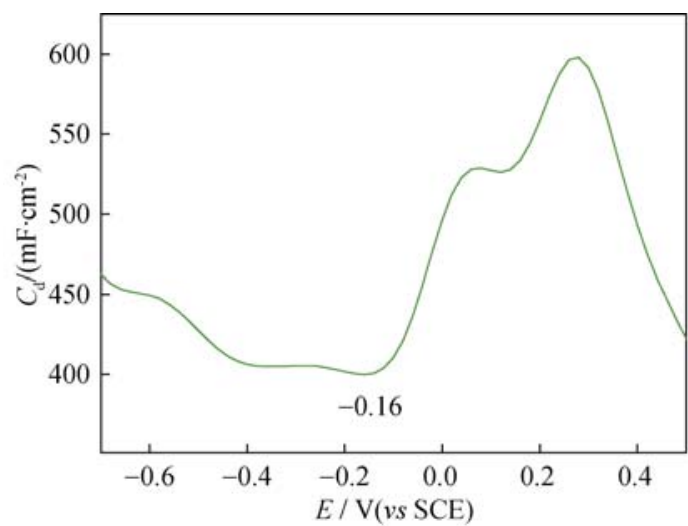

图 $5 \mathrm{Ti} / \mathrm{RuO}_{2}$ 涂层在 $0.001 \mathrm{~mol} / \mathrm{L} \mathrm{NaCl}$ 溶液中微分电容曲线 Fig. 5 Differential capacity curve of $\mathrm{Ti} / \mathrm{RuO}_{2}$ oxide coatings in $0.001 \mathrm{~mol} / \mathrm{L} \mathrm{NaCl}$ solution

\subsection{Tafel 斜率和反应级数}

在 $\mathrm{NaCl}$ 溶液中分别对 $\mathrm{RuO}_{2}$ 和 $\mathrm{Ru}-\mathrm{La}-\mathrm{O}$ 氧化物 涂层做极化曲线, 根据该曲线得到析氯反应动力学 参数列于表 1 。从表 1 可以看到, 加 $\mathrm{La}$ 的氧化物涂 层 $a$ 减小, $i_{0}$ 增大, 过电位 $\eta$ 下降, 表明该电极比纯 $\mathrm{RuO}_{2}$ 具有更好的电化学性能, 这与我们前期的实 验结果相符。 $\mathrm{Ti} / \mathrm{RuO}_{2}$ 和 Ti/Ru-La-O 的 Tafel 斜率分 别为 129 和 $168 \mathrm{mV}$ 。

图 6 为在不同浓度 $\mathrm{NaCl}$ 溶液中测得的 $E$-lgi 关 系曲线, 图中曲线存在近似平行关系, 表明 Tafel 斜 率不随溶液中氯离子浓度发生变化。图 7 是一定电 位下的 $\lg i-\lg c$ 关系图, 根据反应级数的定义:

$$
p=\left(\frac{\partial \ln i}{\partial \ln c_{\mathrm{Cl}^{-}}}\right)_{\eta, T}
$$

因此, 利用图 7 即可求出 $\mathrm{RuO}_{2}$ 和 Ru-La-O 氧化物 涂层的反应级数分别为 0.53 和 0.43 。

Conway 等 ${ }^{[16]}$ 认为应该考虑在浓度较高的溶液中 吸附物间的相互作用, 对于 Volmer-Heyrovsky 机理, 若 考虑吸附物相互作用的影响, 则其动力学方程应为:

$$
i=2 F k_{3} C_{\mathrm{Cl}^{-}} \theta_{\mathrm{Cl}} \exp [+\beta \eta F / \mathrm{RT}] \cdot \exp \left[\beta g \theta_{\mathrm{Cl}}\right]
$$

其中 $k_{3}$ 为反应(3)速度常数, $\beta$ 为对称因子, $g$ 为横向 相互作用参数, 其 Tafel 斜率为:

$$
b=\frac{\mathrm{R} T}{F}\left[\frac{1+g \theta(1-\theta)}{\beta+(1-\theta)(1+g \theta)}\right]
$$

根据该机理得到的反应级数 $p$ 为:

$$
p=1+\frac{[1-\theta(1+\delta)](1-\beta g \theta)}{1+g \theta(1-\theta)}
$$

根据(式 12)和(式 13)式绘制 Tafel 斜率，反应级数 $p$ 与吸附物覆盖度 $\theta$ 的关系曲线如图 8 所示, 这里取 $\beta=0.5$ 。从图 8 可以看到, 当覆盖度 $\theta \rightarrow 0$ 时, $b=2 \mathrm{R} T /$ $3 F=40 \mathrm{mV}, p=2$; 当 $\theta \rightarrow 1$ 时, $b=2 \mathrm{R} T / F=118 \mathrm{mV}$, $p=2-\theta$ 。这表明在所有覆盖度范围内 Tafel 斜率不会 超过 $118 \mathrm{mV}$, 反应级数也不会小于 1 。显然, 根据 Volmer- Heyrovsky 机理不能解释本实验结果。

\begin{tabular}{|c|c|c|c|c|c|c|}
\hline \multirow[t]{2}{*}{ Sample } & \multirow{2}{*}{$\begin{array}{c}a \\
/ \mathrm{V}\end{array}$} & \multirow{2}{*}{$\begin{array}{c}b \\
/ \mathrm{mV}\end{array}$} & \multirow[b]{2}{*}{$p$} & \multirow{2}{*}{$\begin{array}{c}i_{\mathrm{o}} \\
/\left(\mathrm{mA} \cdot \mathrm{cm}^{-2}\right)\end{array}$} & \multicolumn{2}{|c|}{$\eta / \mathrm{V}$} \\
\hline & & & & & $i=1 \mathrm{~mA} / \mathrm{cm}^{2}$ & $i=10 \mathrm{~mA} / \mathrm{cm}^{2}$ \\
\hline $\mathrm{RuO}_{2}$ & 1.098 & 129 & 0.53 & $2.825 \times 10^{-2}$ & 1.216 & 1.388 \\
\hline Ru-La-O & 0.947 & 168 & 0.43 & $4.378 \times 10^{-2}$ & 1.045 & 1.281 \\
\hline
\end{tabular}

如果将吸附 $\mathrm{Cl}$ 在表面氧化膜上再结合作为速 度控制步骤，则在考虑吸附离子相互作用影响的条

表 1 钉镧氧化物涂层析氯反应动力学参数

Table 1 Kinetic parameters of $\mathrm{Ti} / \mathrm{RuO}_{2}$ and $\mathrm{Ti} / \mathrm{Ru}-\mathrm{La}-\mathrm{O}$ oxide coatings for chlorine evolution reaction

$a$ : Tafel line intercept; $b$ : Tafel slope; $p$ : Reaction order with respect to $\mathrm{Cl}^{-} ; i_{0}$ : Exchange current density; $\eta$ : Overpotentials 



图 6 不同浓度下 $\mathrm{Ti} / \mathrm{RuO}_{2}$ (a) 和 Ti/Ru-La-O (b) 氧化物涂层 的 $E$-lgi 关系图

Fig. 6 Potential-current curves of $\mathrm{Ti}^{2} \mathrm{RuO}_{2}$ (a) and $\mathrm{Ti} / \mathrm{Ru}-\mathrm{La}-\mathrm{O}$ (b) oxide coatings for chlorine evolution in various $\mathrm{NaCl}$ solutions from 2 to $5 \mathrm{~mol} / \mathrm{L}$



图 7 氧化物涂层 $\mathrm{Ti} / \mathrm{RuO}_{2}(\mathrm{a})$ 和 Ti/Ru-La-O (b)的 $\lg i-\lg c$ 关系图

Fig. 7 Dependence of the reaction rate for $\mathrm{Ti} / \mathrm{RuO}_{2}$ (a) and $\mathrm{Ti} / \mathrm{Ru}-\mathrm{La}-\mathrm{O}$ (b) oxide coatings on the $\mathrm{Cl}^{-}$concentration

件下, 其动力学方程改为:

$$
i=2 F k_{2} \theta_{\mathrm{Cl}^{*}}^{2} \exp \left[2 g \theta_{\mathrm{Cl}^{*}}\right]
$$

根据该公式得到的 Tafel 斜率和反应级数分别是:

$$
b=\frac{\mathrm{R} T}{F}\left[\frac{1+g \theta(1-\theta)}{2(1+g \theta)(1-\theta)}\right]
$$
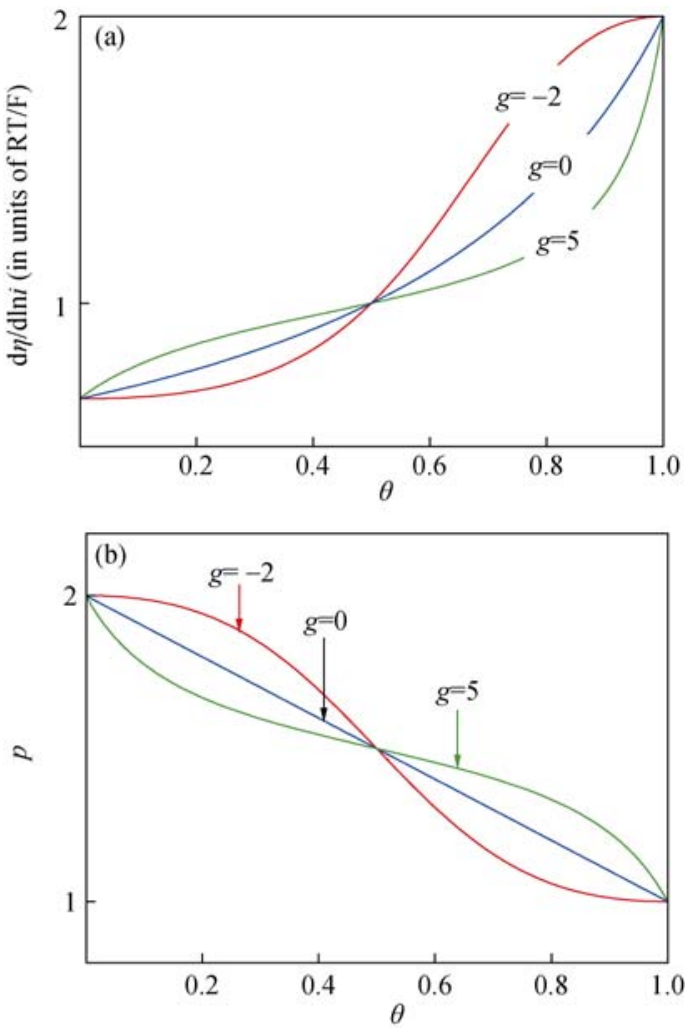

图 8 根据 Volmer-Heyrovsky 机理的 Tafel 斜率与覆盖度 $\theta(a)$ 及反应级数 $p$ 与覆盖度 $\theta(\mathrm{b})$ 在 $g=-2 、 0 、 5$ 时关系曲线

Fig. 8 Relation between Tafel slope and coverage $\theta$ (a), and reaction order $p$ and $\theta$ (b) for Volmer-Heyrovsky mechanism at $g=-2,0,5$

$$
p=\frac{2(1+g \theta)(1-\theta)}{1+g \theta(1-\theta)}
$$

图 9 给出 Volmer-Tafel 下 Tafel 斜率和反应级数 $p$ 与吸附物覆盖度 $\theta$ 的关系曲线。从图中可以看到, 当 $\theta \rightarrow 0$ 时, $b=\mathrm{RT} / 2 F=30 \mathrm{mV}, p=2$; 当 $\theta \rightarrow 1$ 时, $b \rightarrow \infty$, $p \rightarrow 0$ 。根据我们微分电容实验结果, 由于特性吸附 现象的存在导致表面 $\mathrm{Cl}$ *覆盖率很高, 在高电流密 度时 Tafel 斜率上升到较高的值, 因此测出的结果 大于 $118 \mathrm{mV}$, 而反应级数也远低于 1 , 这一结果与 高覆盖度下的 Volmer-Tafel 机理结论相符, 说明析 氯反应是吸附 $\mathrm{Cl}$ *在表面再结合生成 $\mathrm{Cl}_{2}$ 作为反应 过程的控制步骤, 且由于 $\mathrm{La}$ 的加入使表面氯的特 性吸附增强, 导致 Tafel 斜率增大和反应级数的下 降。此外, 从图 9 可以看到, 随着 $\mathrm{Cl}^{*}$ 表面覆盖度的 增加, 吸附物间的相互作用也增大, 引起 Tafel 斜率 迅速增加, 这也是钓针氧化物涂层比纯 $\mathrm{RuO}_{2}$ 涂层 具有更高 Tafel 斜率的原因。

\section{3 结论}

本研究以钉镧氧化物涂层为研究对象, 以 $\mathrm{Ti} / \mathrm{RuO}_{2}$ 

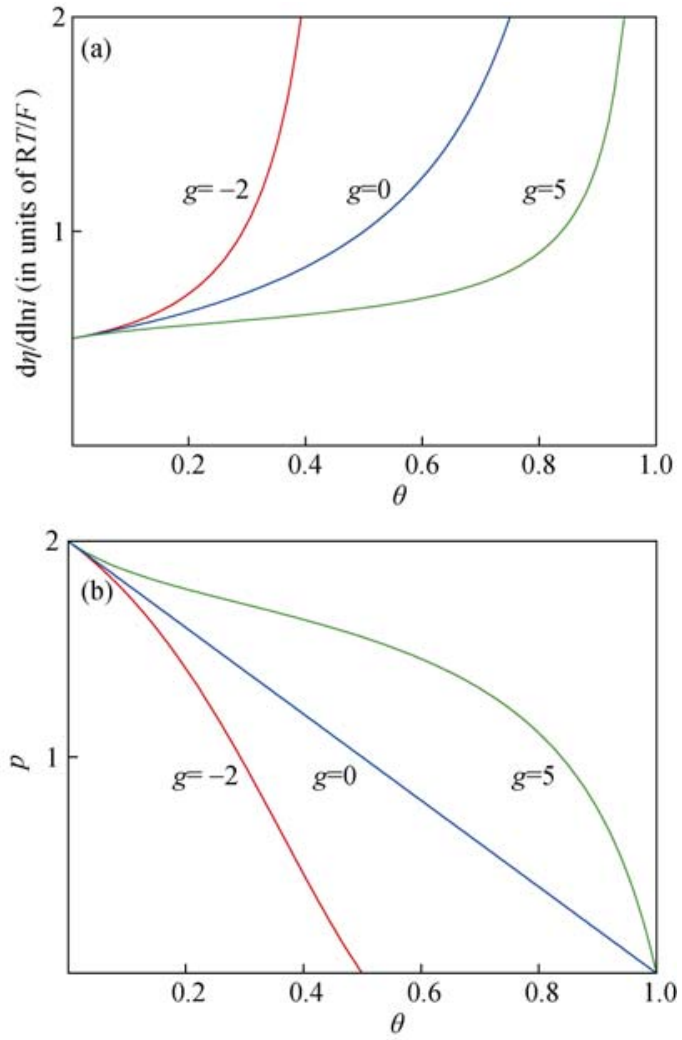

图 9 根据 Volmer-Tafel 机理的 Tafel 斜率与覆盖度 $\theta(a)$ 及反应 级数 $p$ 与覆盖度 $\theta(\mathrm{b})$ 在 $g=-2,0,5$ 时关系曲线

Fig. 9 Relationship between Tafel slope and coverage $\theta$ (a), and reaction order $p$ and $\theta$ (b) for Volmer-Tafel mechanism at $g=-2,0,5$

涂层作为对比，通过微分电容和极化曲线等实验技 术研究了电极涂层表面的特性吸附、La 对析氯反应 的影响及析氯反应的机理, 认为在 $\mathrm{NaCl}$ 的中性溶液 中, 电极表面存在 $\mathrm{Cl}^{-}$的特性吸附, 这种特性吸附对 析氯反应的动力学产生了影响，导致 Tafel 斜率上升, 反应级数下降。加入 $\mathrm{La}$ 使电极的过电位下降, 交换 电流密度增大，这有利于析氯反应的进行。根据实验 数据对 Volmer-Heyrovsky 和 Volmer-Tafel 机理的比 较分析，可以认为，发生该氧化物涂层的析氯反应 符合 Volmer-Tafel 机理过程。

\section{参考文献:}

[1] PETKOV L. Electrooxidation of chloride systems. A review. Oxid. Commun, 2009, 32(3): 654-677.

[2] EXNER K S, ANTON J, JACOB T, et al. Chlorine evolution reaction on $\mathrm{RuO}_{2}(110)$ : ab initio atomistic thermodynamics studypourbaix diagrams. Electrochim Acta, 2014, 120: 460-466.

[3] JANSSEN L J J. The mechanism of the chlorine evolution on different types of graphite anodes during the electrolysis of an acidic $\mathrm{NaCl}$ solution. Electrochim Acta, 1974, 19(6): 257-265.
[4] KUHN A T, MORTIMER C J. The kinetics of chlorine evolution and reduction on titanium-supported metal oxides especially $\mathrm{RuO}_{2}$ and $\mathrm{IrO}_{2}$. J. Electrochem. Soc., 1973, 120(2): 231-236.

[5] JANSSEN L J J, STARMANS L M C, VISSER J G, et al. Mechanism of the chlorine evolution on a ruthenium oxide itanium oxide electrode and on a ruthenium electrode. Electrochim Acta, 1977, 22(10): 1093-1100.

[6] DENTON D A, HARRISON J A, KNOWLES R I. Chlorine evolution and reduction on $\mathrm{RuO}_{2} \mid \mathrm{TiO}_{2}$ electrodes. Electrochim Acta, 1979, 24(5): 521-527.

[7] CONSONNI V, TRASATTI S, POLLAK F, et al. Mechanism of chlorine evolution on oxide anodes study of $\mathrm{pH}$ effects. J. Electroanal. Chem., 1987, 228(1/2): 393-406.

[8] ARDIZZONE S, CARUGATI A, LODI G, et al. Surface structure of ruthenium dioxide electrodes and kinetics of chlorine evolution. J. Electrochem Soc., 1982, 129(8): 1689-1693.

[9] JANSSEN L J J, VISSER G J, BARENDRECHT E. Effect of molecular chlorine diffusion on theoretical potential-current density relations for chlorine evolving electrode. Electrochim Acta, 1983, 28(2): 155-163.

[10] ZHANG S J, DU A L, XU L K, et al. Study on deactivation behavior of metal oxide anode at differernt seawater electrolysis temperature. Rare Metal Mat. Eng., 2013, 42(12): 2613-2618.

[11] BURKE L D, O'NEILL J F. Some aspects of the chlorine evolution reaction at ruthenium dioxide anodes. J. Electroanal. Chem., 1979, 101(3): 341-349.

[12] FAITA G, FIORI G. Anodic discharge of chloride ions on oxide electrodes. J. Appl. Electrochem., 1972, 2(1): 31-35.

[13] ERENBURG R G. Mechanism of the chlorine reaction of rutheniumtitanium oxide anodes. Soviet Electrochemistry, 1984, 20(12): $1481-1486$.

[14] KRISHTALIK L I. Kinetics and mechanism of anodic chlorine and oxygen evolution reactions on transition metal oxide electrodes. Electrochim Acta, 1981, 26(3): 329-337.

[15] AUGUSTYNSKI J, BALSENC L, HINDEN J. X-Ray photoelectron spectroscopic studies of $\mathrm{RuO}_{2}$-based film electrodes. J. Electrochem. Soc., 1978, 125(7): 1093-1097.

[16] CONWAY B E, NOVAK D M. Chloride ion adsorption effects in the recombination-controlled kinetics of anodic chlorine evolution at Pt electrodes. Journal of the Chemical Society, Faraday Transactions 1, 1979, 75: 2454-2472.

[17] KUSMIEREK E, CHRZECIJANSKA E. Use of the $\mathrm{CeO}_{2}$-modified $\mathrm{Ti} \mathrm{TiO}_{2}-\mathrm{RuO}_{2}$ electrodes in oxidation of acid orange 7. Przem. Chem., 2012, 91(10): 2038-2043.

[18] GAN W P, LIU J Y, LIU H, et al. Characterization and electrochemical properties of $\mathrm{RuO}_{2}$ film electrode on the inner-wall of tantalum shell for capacitor. J. Inorg. Mater., 2010, 25(8): 
882-886.

[19] SUN J M, WANG X, WEI Z P, et al. Effects of sintering temperature on $\mathrm{Ti} / \mathrm{RuO}_{2}-\mathrm{CeO}_{2}$ electrodes applied in super-capacitors. $J$. Chin. Rare Earth Soc., 2011, 29(6): 718-723.

[20] NOWAKOWSKI P, VILLAIN S, AGUIR K, et al. Microstructure and electrical properties of $\mathrm{RuO}_{2}-\mathrm{CeO}_{2}$ composite thin films. Thin Solid Films, 2010, 518(10): 2801-2807.

[21] FAN N J, ZHU P X, ZHOU S G, et al. Optimization for preparation technique of Ru-La oxide coating titanium anode by orthogonal design. Hot Working Technology, 2014, 43(12): 160-162.

[22] MURAKAMI Y, KONDO T, SHIMODA Y, et al. Effects of rare earth chlorides on the preparation of porous ruthenium oxide electrodes. J. Alloys Compd., 1996, 239(2): 111-113.

[23] LONG P, XU L K, LI Q F, et al. Effect of microstructure on electrochemical activity of Ru-La oxide coatings on Ti substrate. Rare Metal Mat. Eng., 2013, 42(6): 1185-1189.

[24] LONG P, LI Q F, XU L K, et al. Electrochemical impedance spectroscopy (EIS) study of Ru-La oxide coatings in $\mathrm{NaCl}$ solution. Acta Chim. Sinica, 2012, 70(10): 1166-1172.

[25] LONG P, XU L K, XUE L L, et al. Voltammetry behavior of $\mathrm{Ru}-\mathrm{La}$ oxide coatings on Ti substrate in $\mathrm{NaCl}$ solution. Rare Metal
Mat. Eng., 2014, 43(5): 1133-1137.

[26] BURROWS I R, DENTON D A, HARRISON J A. Chlorine and oxygen evolution on various compositions of $\mathrm{RuO}_{2} \backslash \mathrm{TiO}_{2}$ electrodes. Electrochim Acta, 1978, 23(6): 493-500.

[27] VALETTE G, PARSONS R. Adsorption on well-defined solid surfaces chloride adsorption on a (110) face of silver. J. Electroanal. Chem., 1986, 204(1/2): 291-297.

[28] DAGHETTI A, LODI G, TRASATTI S. Interfacial properties of oxides used as anodes in the electrochemical technology. Mater. Chem . Phys., 1983, 8(1): 1-90.

[29] 阿伦· $\cdot$ ·巴德 美, 拉里·r·福克纳. 电化学方法: 原理和应用. 第 二版. 北京: 化学工业出版社, 2005: 571 .

[30] NOVAK D M, CONWAY B E. Competitive adsorption and state of charge of halide ions in monolayer oxide film growth processes at Pt anodes. Journal of the Chemical Society, Faraday Transactions 1: Physical Chemistry in Condensed Phases , 1981, 77: 2341-2359.

[31] TAKASU Y, ARIKAWA T, YANASE K, et al. Preparation of a novel Pt-RuO ${ }_{2} \backslash \mathrm{Ti}$ electrocatalyst by use of highly porous ruthenium oxide support prepared from $\mathrm{RuO}_{2}-\mathrm{La}_{2} \mathrm{O}_{3}$ /electrode. J. Alloys Compd., 1997, 261(1/2): 172-175. 